\title{
Carinthie-Slovénie : un espace économique et humain à reconstruire
}

Carinthia-Slovenia : an economical and human space to be rebuilt

Kärnten-Slowenien : Der steinige Weg zur wirtschaftlichen und sozialen

Kooperation

\section{Brigitta Busch et Heike Schiebeck}

\section{(2) OpenEdition}

\section{Journals}

\section{Édition électronique}

URL : http://journals.openedition.org/rge/2300

DOI : $10.4000 /$ rge.2300

ISSN : 2108-6478

Éditeur

Association des géographes de l'Est

Édition imprimée

Date de publication : 1 septembre 2003

ISSN : 0035-3213

Référence électronique

Brigitta Busch et Heike Schiebeck, « Carinthie-Slovénie : un espace économique et humain à reconstruire ", Revue Géographique de l'Est [En ligne], vol. 43 / 4 | 2003, mis en ligne le 24 novembre 2010, consulté le 08 septembre 2020. URL : http://journals.openedition.org/rge/2300 ; DOI : https:// doi.org/10.4000/rge.2300

Ce document a été généré automatiquement le 8 septembre 2020.

Tous droits réservés 


\section{Carinthie-Slovénie : un espace économique et humain à reconstruire}

Carinthia-Slovenia : an economical and human space to be rebuilt

Kärnten-Slowenien : Der steinige Weg zur wirtschaftlichen und sozialen

Kooperation

Brigitta Busch et Heike Schiebeck

1 Bien que la coopération transfrontalière soit aujourd'hui à l'ordre du jour, avec l'élargissement de l'UE à l'Est, elle se heurte à de nombreux obstacles. L'article suivant traite des communes frontalières d'Eisenkappel- Vellach / Zelezna Kapla-Bela, Jezersko et Solcava (Fig. 1). Après une description des caractéristiques démographiques et socio-économiques de ces espaces périphériques, il analyse dans une perspective historique les réticences mentales et la construction des mythes autour de la frontière, qui continuent à empêcher le développement de l'espace transfrontalier en Carinthie. L'étude d'un projet du programme Interreg Carinthie-Slovénie montre comment de petites initiatives associatives ont réussi à réaliser leur coopération transfrontalière. 
Figure 1 : La région frontalière d'Eisenkappel

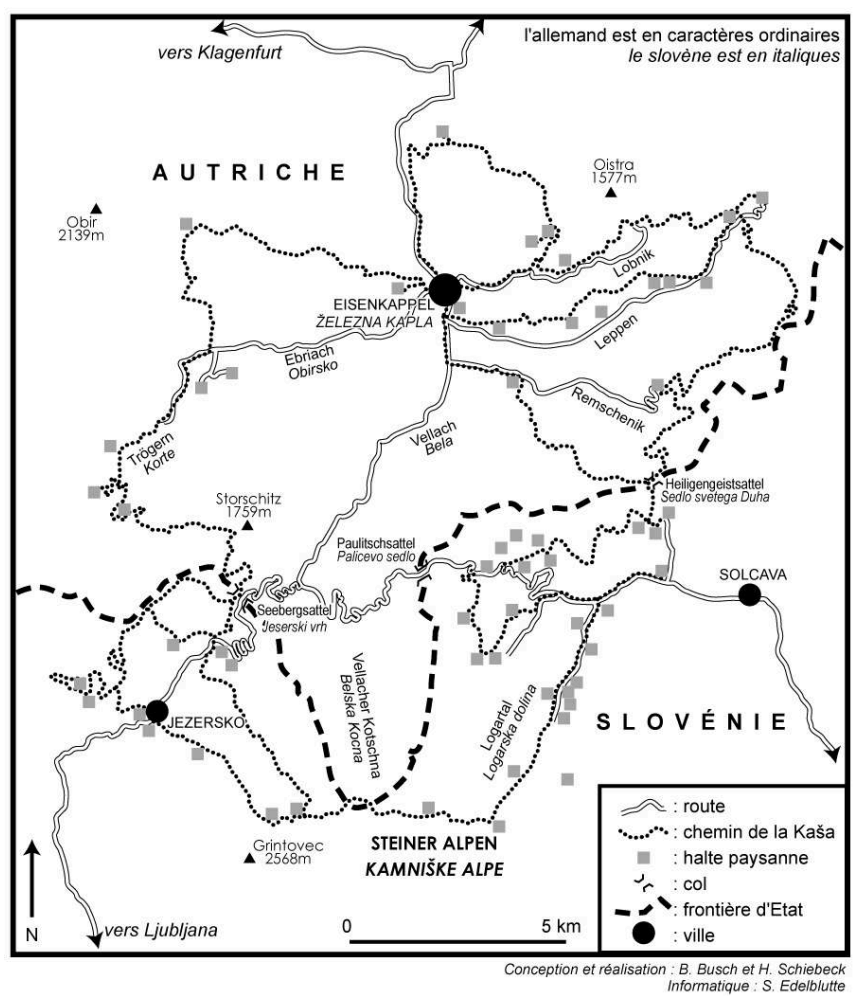

\section{L'exemple d'Eisenkappel-Vellach / Zelezna kapla- Bela : une commune à l'ombre de la frontière}

2 La commune de Eisenkappel-Vellach / Zelezna Kapla-Bela, dans le district de Völkermarkt, est située dans la partie la plus méridionale de l'Autriche, dans les Alpes calcaires. Elle s'étend sur $198,9 \mathrm{~km}^{2}$ et compte parmi les communes les plus étendues du Land de Carinthie. Son territoire est entouré sur plus de la moitié par la frontière avec la Slovénie. Une partie de la population est bilingue (allemand / slovène) : au recensement de 2001, $39 \%$ de ses habitants déclarent utiliser couramment le slovène (Statistik Austria).

3 On peut se rendre au pays voisin par deux cols : la route du Seeberg et, depuis avril 2000, celle du Paulitsch. La position du col du Seeberg, qui ouvre l'accès à la plaine, puis à la mer, a fait d'Eisenkappel, déjà au moyen âge, un lieu de commerce et de marché important sur la route du sel, nommé Coppel (Ostravsky, 1998). Le commerce du sel et du fer ${ }^{1}$ a attiré une bourgeoisie germanophone, installée dans le bourg, et qui s'est peu à peu enrichie. Elle défendait ses privilèges contre les habitants des montagnes alentour, majoritairement slovénophones. Dès le début du XVIIe siècle, les bourgeois de Coppel ont mené une lutte acharnée contre l'installation de " ceux venus d'en-haut ». Quelques-uns ont été tolérés, très peu d'entre eux acceptés comme bourgeois (Busch, 1996). Les paysans étaient interdits de commerce et toute infraction était punie de lourdes amendes. Le clivage bourg/montagne est aujourd'hui encore perceptible.

En 2001, la commune comptait 2710 habitants (Statistik Austria). Un peu plus d'un tiers de la population vit dans le bourg d'Eisenkappel, qui s'étire au fond d'une vallée encaissée, où convergent une série d'étroites vallées transversales. Les villages, 
hameaux et fermes de ces vallées et des montagnes alentour constituaient jusqu'en 1963 la commune autonome de Vellach. C'est là, dans des fermes isolées, à une altitude de 600 à $1200 \mathrm{~m}$, que vivent les deux tiers de la population. Les paysans de la montagne tirent aujourd'hui leurs revenus principalement de l'exploitation de la forêt, qui recouvre $83 \%$ du territoire de la commune.

5 L'évolution démographique de la commune d'Eisenkappel-Vellach (Fig. 2) est étroitement liée au commerce du sel et du fer, et plus tard à l'usine de cellulose Rechberg-Obir. La population, qui avait commencé à baisser à partir de 1869 avec le déclin de l'activité commerciale, augmente dès l'ouverture de l'usine en 1890. Les années suivant la Première Guerre mondiale connaissent à nouveau une diminution. La fin de l'Empire austro-hongrois et l'établissement de la frontière en 1920 fait passer Eisenkappel à la périphérie : tout un espace économique vieux de plusieurs siècles est coupé en deux, des liens sociaux et culturels sont rompus. Dans les années 30, malgré la crise économique mondiale et le chômage, les gens trouvent du travail à l'usine et dans les fermes; ils retournent vivre à la campagne, et la population se remet à augmenter. Après la Deuxième Guerre mondiale, elle atteint son point culminant avec 1000 emplois dans l'usine en 1951. La mécanisation supprime des emplois après 1959. En 1979, après un an de fermeture, l'usine est rachetée par l'industrie slovène de papier "Slovenija papir ». Gérée de l'autre côté de la frontière, elle assure l'écoulement de la cellulose en Slovénie et des emplois en Autriche. Cette coopération transfrontalière est brutalement interrompue quand le gouvernement du Land de Carinthie, dirigé à l'époque par Jörg Haider, refuse de contribuer financièrement à la construction d'une station d'épuration pour laquelle le propriétaire slovène et le gouvernement de Vienne sont prêts à s'engager. Avec la nouvelle fermeture de l'usine Rechberg-Obir en 1989, 220 emplois disparaissent. Pendant un siècle, l'usine a été le nerf vital de la vallée, en procurant un travail d'appoint et de proximité aux paysans, tout en leur achetant le bois. Désormais leur survie est menacée à terme. De nombreux habitants de Vellach considèrent la fermeture comme une décision politique contre la minorité slovène en Autriche et contre la volonté slovène de rétablir un espace économique transfrontalier. La disparition d'autres entreprises dans les années qui suivent accentue un exode rural qui n’a jamais cessé depuis (Rechberg-Obir, 1995). 
Figure 2 : Évolution démographique : la commune d'Eisenkappel-Vellach (Gemeinde) en comparaison avec le district de Völkermarkt (Bezirk) et le Land de Carinthie (Bundesland)

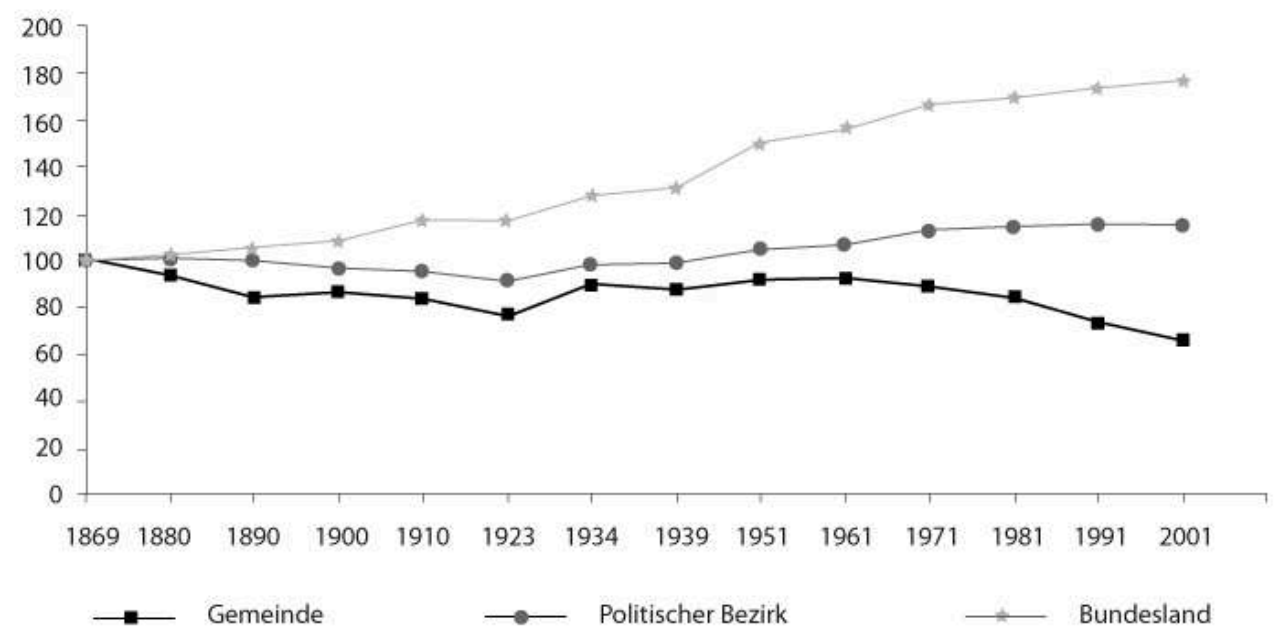

www.statistik.at

6 La commune d'Eisenkappel-Vellach possède les caractéristiques des régions frontalières de l'est et du sud-est de l'Autriche: ce sont des régions agricoles; l'artisanat et l'industrie sont concentrés dans quelques entreprises, surtout dans des branches à bas salaires; les emplois manquent ; seuls les emplois féminins sous-payés dominent; de plus en plus de gens font la navette pour aller travailler ailleurs, de plus en plus loin ; la population émigre (Krajasits 1998). Le nombre de résidents travaillant au-dehors a quasiment triplé de 1971 à 1991. Après la fermeture de l'usine de cellulose le nombre de personnes partant travailler la semaine entière a considérablement augmenté. En 1991, sur 1195 personnes actives résidant à Eisenkappel-Vellach, plus de la moitié part travailler ailleurs (Statistik Austria) ${ }^{2}$.

7 En 1999, la commune compte 176 exploitations pratiquant l'agriculture d'élevage et le forestage ; pour 48 d'entre elles c'est l'activité principale, pour 121 l'activité secondaire, 7 sont des personnes juridiques. La surface moyenne des fermes de la première catégorie est d'environ 150 ha et de 58 ha pour la seconde, avec seulement une surface moyenne cultivable de moins de 10 ha, le reste étant de la forêt difficile d'accès. L'agriculture autrichienne dans son ensemble est caractérisée par de petites exploitations, surtout en montagne; plus de la moitié ont moins de 10 ha de surface agricole utile, ce qui est bien en-dessous de la moyenne d'autres États de l'UE. En 1997, pour l'ensemble des exploitations, elle est en Autriche de 16,3 ha, en France de 41,7 ha et en Grande-Bretagne de 69,3 ha (Eurostat, 1997). Avec une surface agricole utile de 5,6 ha par ferme, l'agriculture en Slovénie - qui n'a presque pas été collectivisée sous le socialisme - est encore plus morcelée qu'en Autriche. La politique agricole menée par ces deux pays a toujours été en faveur du maintien des petites et moyennes exploitations de montagne. Dans les années 70, le gouvernement autrichien avait classé $80 \%$ du territoire national en zones défavorisées, bénéficiant d'aides directes spécifiques. Ce système est actuellement repris par la Slovénie. Avec l'entrée de l'Autriche dans l'UE, les aides directes ont été remplacées par un système compliqué et bureaucratique de primes de compensation par hectare et par tête de bétail. Aujourd'hui, l'effet de la répartition des aides est inverse: les primes les plus 
importantes vont aux grandes exploitations de plaine avec le plus grand nombre d'hectares et de bêtes (Krammer 1995).

En Autriche, 5,2 \% de la population active travaille dans l'agriculture. Le recensement de 1991 (Statistik Austria) donne pour Eisenkappel 143 personnes actives dans l'agriculture et le forestage, c'est-à-dire un quart des habitants travaillant sur place. L'enquête menée depuis 1995 en Autriche sur les structures agricoles selon les critères de l'UE (Statistik Austria) donne des chiffres très différents : pour l'année 1999, 428 personnes ont déclaré travailler dans l'agriculture, donc trois fois plus que lors du recensement national. Cette différence s'explique du fait que les personnes recensées ont estimé que le travail signifiait prioritairement un travail salarié, tandis que l'enquête sur les structures agricoles a considéré comme actives dans l'agriculture toutes les personnes âgées de plus de 16 ans participant à l'exploitation de la ferme. Ceci montre bien l'importance des activités de subsistance non-rémunérées qui assurent la pérennité de l'exploitation. Elles n'apparaissent pas dans le cadre du recensement parce qu'elles ne sont pas un travail reconnu comme tel dans la société. Dans ses « Études paysannes » le sociologue Teodor Shanin définit l'économie paysanne comme une économie spécifique liée à la survie. "C'est une économie qui tend à assurer la survie plutôt que l'accumulation de capitaux, à optimiser le fruit du travail et non le profit " (Shanin, 2000)3. Ces formes d'économie solidaire prennent aujourd'hui de plus en plus d'importance, même si elles sont jugées marginales par le système actuel. "La paysannerie ne cadre avec aucun des concepts de la société contemporaine »(Shanin, 1990). La vie d'Eisenkappel-Vellach est fortement imprégnée par cette économie paysanne. Presque tous ses habitants ont un rapport avec elle.

Depuis 1989, la commune a entrepris des efforts pour créer des emplois. Le développement du tourisme de passage a donné du travail un temps aux entreprises de bâtiment, comme l'aménagement de la grotte de stalactites de l'obir. Doris Herzog et Doris Wastl-Walter tirent un bilan positif de la nouvelle orientation : dans leur travail sur «la restructuration économique d'Eisenkappel / Zelezna Kapla», elles analysent d'après les statistiques le passage de l'activité industrielle au tourisme. Les problèmes qui ont surgi après la fermeture de l'usine de cellulose auraient été résolus par un changement radical d'orientation de l'économie locale, changement qui a surtout bénéficié à la population du bourg et à ses commerçants, tandis que les habitants des vallées de montagne étaient encore oubliés: "Ceux qui étaient le plus touchés par la fermeture [comme les paysans] n'ont pas directement profité de la restructuration. A la place d'emplois d'ouvriers spécialisés dans la cellulose, on a créé des emplois féminins dans le tourisme et la station thermale; seuls quelques chômeurs ont été embauchés comme manœuvres " (Herzog, Wastl-Walter, 1995).

10 Non seulement les paysans d'Eisenkappel-Vellach ont perdu leur activité d'appoint et l'écoulement de leur bois, mais l'entrée de l'Autriche dans l'UE représente pour eux une nouvelle menace : les prix à la production baissent, tandis qu'ils doivent investir pour mettre leur exploitation aux normes européennes. Pour y faire face, ils réfléchissent aux moyens de valoriser leurs productions traditionnelles, dans une démarche collective. C'est ainsi que nait l'idée d'un groupement d'entraide pour le maintien des fermes de montagne, qui prendra le nom de Coppla Kasa ${ }^{4}$. 
Figure 3 : Le sigle de l'association « Coppla Kasa ».

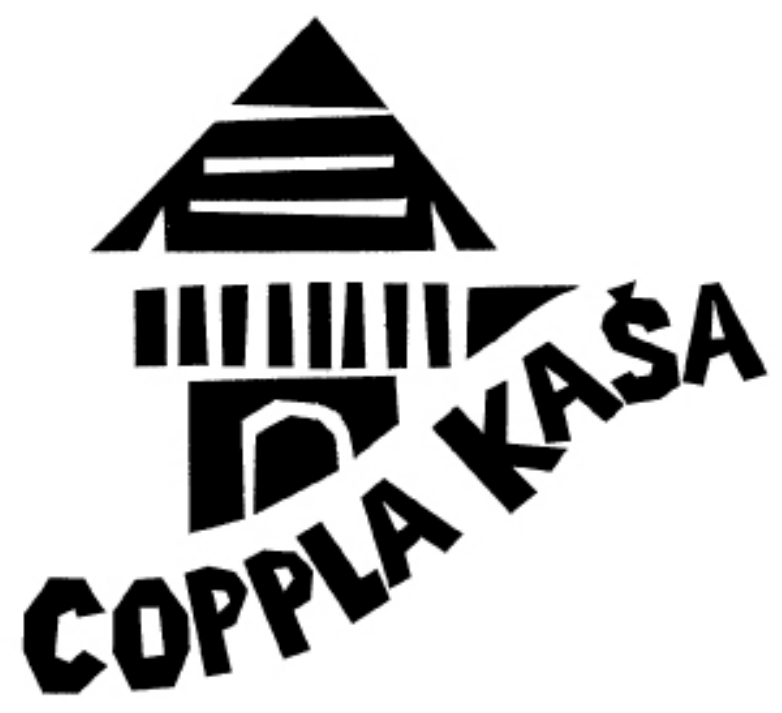

\section{La frontière, une barrière mentale}

Dans une étude comparative entre les régions du sud et du sud-est de l'Autriche limitrophes aux anciens pays socialistes, Cornelia Krajasits (1998) conclut que les communes de la Carinthie du Sud sont celles qui, du point de vue économique, ont le moins profité des changements survenus depuis 1989. Ceci malgré le fait que dans le passé, la frontière avec la Yougoslavie était déjà plus perméable que celle avec la Hongrie ou la Tchécoslovaquie, et malgré l'existence d'une population partiellement bilingue. Krajasits dénonce les réticences liées aux "frontières dans la tête » comme étant les principaux obstacles à la coopération transfrontalière. Cette conclusion est soulignée par Hans-Joachim Bodenhöfer (2000) dans son étude pour le syndicat autrichien des patrons. Selon lui, l'intégration (de la Slovénie dans l'UE) pourrait se faire en passant par-dessus la Carinthie - vu le manque d'ouverture et d'intérêt pour l'adhésion prochaine, et la persistance des barrières mentales.

Les interviews réalisées dans le cadre du projet européen «Border identities » (voir éditorial dans ce numéro) sur le thème de l'élargissement de l'UE confirment l'existence de ces barrières. "La seule chose que j'approuve dans l'UE, ce serait pour moi l'ouverture des frontières et la monnaie commune. (... ) Cela me plairait bien d'aller où je veux et de payer avec la même monnaie et que les frontières soient ouvertes. (... ) Ce serait beau. Les frontières ouvertes en soi. Tout de même, je dois dire franchement, quand je pense à toutes les canailles qui vivent dans ces pays en voie de développement, à tous ces filous, bon, en principe, je dirais pas tous, mais vraiment beaucoup sont très paresseux et ils veulent avoir en une année ce que nous avons mis quarante ans à acquérir. Ça je ne peux pas le comprendre, et dans ce cas il est sans doute préférable que la frontière reste fermée.» Cet extrait de l'interview d'une Autrichienne habitant la commune d'Eisenkappel souligne une ambiguité typique dans les déclarations de nombreuses personnes interrogées: on approuve l'ouverture imminente de la frontière austro-slovène au niveau personnel - passage sans contrôle de papiers et sans formalités douanières - d'autant plus que beaucoup de gens ont de la famille ou possèdent des terrains de l'autre côté. Mais en même temps, chez les 
Autrichiens, on craint la perte d'une certaine fonction protectrice attribuée aux frontières par rapport aux « autres ", « ceux du sud ».

D'où viennent ces réticences, ces barrières mentales empêchant le développement d'une coopération transfrontalière, révélées autant par les propos des habitants que par l'analyse des données économiques?

14 La frontière actuelle entre l'Autriche et ses voisins du Sud est tracée sur la carte politique depuis 1920. Mais la situation en Carinthie est déjà polarisée depuis longtemps. Lors de leur formation dans la deuxième moitié du XIXe siècle, les partis politiques se sont démarqués selon des affiliations linguistiques et ethniques. Le parti conservateur catholique slovène représentant les paysans et le clergé d'un côté, le parti libéral nationaliste allemand représentant les marchands, les patrons et le personnel administratif de l'autre. Le mouvement ouvrier naissant se range majoritairement côté allemand, bien que recrutant beaucoup de ses partisans parmi la population rurale de langue slovène. En fait, les divisions ethniques, linguistiques, sociales et politiques coïncident largement, d'où l'existence d'une frontière imaginaire dans le discours public. Elle est définie comme une frontière linguistique, localisée et identifiée en tant que ligne de séparation. On organise même des excursions à la manière d'une inspection de front militaire (Judson, 2001). Les villages bilingues de Carinthie sont perçus par les nationalistes allemands comme les derniers bastions du monde germanique face au monde slave.

15 Après la Première Guerre mondiale et l'éclatement de l'Empire austro-hongrois, le tracé de la frontière entre les deux nouveaux États, la République autrichienne et le Royaume des Serbes, Croates et Slovènes, devient l'objet de conflits armés. Lors d'un référendum organisé par la Société des Nations dans la région concernée, la population, dont un nombre considérable de slovénophones, opte majoritairement pour l'appartenance à la République. Ces événements sont à l'origine du mythe de la frontière "écrite par le sang" célébré chaque année par des défilés en costume national dans un grand déploiement de drapeaux carinthiens. Ce mythe est forgé de deux éléments : se défendre contre la menace slave venant du Sud et préserver «la Carinthie libre et indivisible ». Il évoque une homogénéisation à l'intérieur et une démarcation vis-à-vis des Balkans, représentant «l'autre» fondamentalement différent. Dans son livre célèbre «L'invention des Balkans » (Die Erfindung des Balkans, 1997) Maria Todorova explique que les Balkans ont servi de décharge sauvage pour toutes sortes de caractéristiques négatives à partir desquelles on a créé à l'inverse une image flatteuse de l'Europe et de l'Ouest. Tout en faisant partie de l'Europe, les Balkans sont placés dans le rôle d'un alter ego sur lequel sont extériorisées et projetées les tensions et contradictions internes.

16 État souverain depuis 1991 seulement, la Slovénie base depuis le XIX ${ }^{\mathrm{e}}$ siècle son mythe nationalsur la culture, celle-ci étant comprise comme synonyme de littérature et langue (Velikonja, 1996). La nation slovène comprend donc tous les slovénophones dispersés sur plusieurs États. L'histoire slovène est vue comme une succession de soumissions à des entités politiques étrangères. Le mythe du "bon vieux temps " évoquant un ordre social juste joue un rôle significatif, avec au centre le paysan idéalisé comme antinomie à la «bourgeoisie déracinée " et au "prolétariat apatride " (Velikonja, 1996). Comme la minorité slovène en Autriche est jusque dans les années 1980 liée à la vie agricole, elle peut servir aux Slovènes des centres urbains à projeter leurs rêves bucoliques évoquant un passé archaïque. À cela s'ajoute un sentiment 
paternaliste en faveur de la minorité discriminée, qui la confine au folklore et la prive de son rôle d'acteur politique.

Le mythe national carinthien, comme le slovène, a subi certaines transformations au cours de l'histoire, mais il persiste dans ses fondements et reste présent autant dans les récits personnels collectés dans le cadre du projet «Border Identities » que dans les discours politiques. Il n'est donc pas étonnant que la minorité slovène - malgré les nombreux liens personnels que la frontière de 1920 n'a pas réussi à rompre complètement - n'ait guère pu exercer une fonction active dans une coopération transfrontalière.

En 1938, l'Autriche devient une partie de l'Allemagne nazie, et lorsque Hitler attaque la Yougoslavie en 1941, une grande partie de la Slovénie est également annexée au Reich. Les frontières sont déplacées vers le sud afin de germaniser la Carinthie ainsi que les régions annexées en Slovénie. Des deux côtés de l'ancienne frontière, les Slovènes sont déportés par milliers et vont grossir les rangs de la résistance organisée par l'armée de Tito. En 1945, les forces yougoslaves et britanniques libèrent simultanément la Carinthie. La coopération initiale entre les deux alliés ne dure pas, le combat contre le communisme devenant la priorité de la politique étrangère britannique. Après la guerre, les frontières sont rétablies suivant la ligne d'avant 1938. Jusqu'en 1949, la République Socialiste Yougoslave maintient - comme dans le cas de Trieste - des revendications territoriales à l'encontre de la zone bilingue de Carinthie.

Entre 1945 et 1949, la frontière austro-yougoslave reste hermétiquement fermée. Elle devient ligne de séparation entre deux systèmes politiques et idéologiques. Selon Étienne Balibar (1997), « aucune frontière politique n'est jamais la simple limite entre deux États, mais toujours surdéterminée, et en ce sens à la fois sanctionnée, redoublée et relativisée par d'autres divisions géopolitiques. » Balibar cite explicitement l'exemple de la guerre froide. Dans la logique des blocs, « les frontières nationales d'États ont été à nouveau surdéterminées et, selon les cas, renforcées ou affaiblies». Cette surdétermination géopolitique reste valable pour la frontière entre l'Autriche et la Yougoslavie, même lorsqu'en 1949 cette dernière quitte la sphère d'influence soviétique. La frontière entre les deux États sera moins hermétique que le rideau de fer, mais tout de même moins perméable que les frontières autrichiennes avec les pays de l'Ouest.

La présence de troupes yougoslaves en 1945 - bien que limitée à quelques jours - et les revendications territoriales yougoslaves permettent de revitaliser le mythe national carinthien de la défense de la patrie et de la "frontière naturelle » le long de la crête des Alpes calcaires du Sud. Il est transformé du fait qu'au danger slave s'ajoute celui du communisme. Par conséquent, les Slovènes de Carinthie qui avaient largement soutenu la résistance armée des partisans sont stigmatisés comme étant la " $5^{\mathrm{e}}$ colonne" ennemie - sauf s'ils préfèrent renier leur origine et s'assimiler. L'Autriche d'aprèsguerre forge son identité sur "le mythe d'un nouveau départ, la Stunde Null (heure zéro), basé sur une attitude de pitié de soi-même et de rejet collectif des responsabilités", se proclamant "victime du nazisme - et ensuite des forces alliées occupantes» (Wodak et al., 1999). S'y ajoute un autre élément, celui « des acquis économiques et sociaux de la Deuxième République, en particulier l'État-providence et la paix sociale et économique (en opposition à l'ex-Yougoslavie, à l'Europe de l'Est et à d'autres États)» (Wodak et al., 1999). Ce discours contribue à alimenter ou réactualiser l'ancien sentiment de supériorité de la race germanique par rapport aux Slaves. 
21 Le Traité d'État de 1955 conclu avec les Forces Alliées rétablit l'Autriche comme État souverain. En Carinthie, les décennies suivantes sont marquées par une série de conflits concernant la mise en application des droits octroyés par le Traité d'État à la minorité slovène. En 1972 éclate ce qu'on appellera la "guerre des panneaux »: lorsque le gouvernement de Vienne fait poser des panneaux topographiques bilingues, une foule excitée par des cercles nationalistes allemands les arrache aussitôt. Pendant des années les communes de Carinthie du Sud resteront sans panneaux et jusqu'à nos jours la question n'est toujours pas résolue. La charge émotive extrême de cette question s'explique par le rapport intime entre langue et territoire. Étienne Balibar souligne « qu'il n'y a pas d'autre moyen de réaliser la frontière comme séparation absolue, que de la représenter comme une frontière religieuse - y compris lorsque cette religion est une religion laïque, sécularisée, religion de la langue, de l'école ou du principe constitutionnel » (Balibar, 1997). Le fait de vouloir "marquer » la zone bilingue en Carinthie à l'aide de signes topographiques est ressenti comme une menace à l'intégrité territoriale protégée par la frontière d'État, comme une tentative de déplacer la borne entre "nous » et «les autres ».

22 Alors que la Yougoslavie joue son rôle de protecteur de la minorité slovène, une contradiction apparaît entre le pouvoir fédéral de Vienne, qui cherche un rapprochement avec la Yougoslavie, et les autorités de Carinthie. Ces dernières désignent la minorité slovène comme "un obstacle aux relations de bon voisinage » (Valentin, 1997) et gèlent les rapports avec le pays voisin. Par contre, à partir des années 1980, la "petite" circulation transfrontalière se développe: des Slovènes viennent faire leurs achats en Carinthie, des Autrichiens font le plein d'essence en Slovénie. En même temps la Slovénie commence à s'orienter de plus en plus vers l'Europe de l'Ouest, surtout sur le plan économique.

En 1990, le Parlement de Ljubljana déclare l'indépendance de la Slovénie. Suivent pendant quelques jours des incidents armés entre l'Armée populaire yougoslave et la Défense territoriale slovène. L'objet principal du conflit porte sur le contrôle des frontières : qui doit s'en charger? L'Autriche envoie de son côté des forces armées pour veiller à l'intégrité de sa frontière et les sympathies vont clairement à la petite Slovénie. Avec la reconnaissance internationale de la Slovénie comme État souverain, la désintégration de la Yougoslavie et, plus généralement, la fin de la bipolarité de l'Europe, la frontière austro-slovène perd sa connotation de ligne séparant deux systèmes politiques. En Slovénie, un nationalisme "modéré » occupe la place du vide laissé par la disparition de l'idéologie collectiviste autogestionnaire. Dans une telle situation «seul un type d'appartenance capable d'identifier les membres du groupe de manière plus ou moins automatique »(Puhovski, 2001) peut prendre la relève. Dans la première euphorie suivant l'indépendance, le rôle du nouvel État comme "mère-patrie " pour tous les Slovènes, en incluant ceux vivant au-delà des frontières territoriales et dans le monde entier, est mis en avant. On souligne l'appartenance millénaire à la culture occidentale pour se démarquer des Balkans. Dans le discours politique, la nouvelle frontière au sud est considérée comme une frontière réelle car culturelle, alors que les frontières avec l'Autriche et l'Italie sont quasiment ignorées.

24 Avec l'adhésion de l'Autriche à l'Union Européenne en 1995, la frontière austro-slovène devient frontière extérieure de l'Union et de l'espace Schengen. Alors que les contrôles douaniers sont adoucis côté slovène, ils deviennent de plus en plus stricts côté autrichien. Comme l'Autriche quelques années auparavant, la Slovénie qui souhaite 
adhérer le plus rapidement possible à l'UE anticipe les critères du Traité de Schengen : elle commence à renforcer la surveillance de sa frontière avec la Croatie en dressant un deuxième cordon sécuritaire de la Forteresse Europe. Et, comme en Autriche, le thème de l'immigration illégale devient un leitmotiv du discours politique et médiatique comportant tous les stéréotypes attribués aux « autres » à « ceux du Sud ».

Des deux côtés de la frontière surgit un nouveau discours politique, que l'on retrouve également dans les récits personnels des personnes interrogées, basé sur trois éléments : l'affirmation d'un héritage commun, la projection d'une future appartenance comme petit pays à la communauté de destin européenne et la désignation d'un troisième "autre ». Cela permet de trouver un terrain commun en dépassant les clivages historiques, grâce à l'invention d'une nouvelle frontière mentale déplacée vers le Sud (Hipfl et al., 2002).

Côté carinthien, ce processus se développe non sans ambiguïté. L'idée de former avec les voisins en Italie et en Slovénie une grande Région transfrontalière est considérée tantôt comme une chance, tantôt comme une menace. Les cercles traditionalistes allemands prétendent que l'identité nationale serait en danger d'être noyée dans un amalgame culturel. La "slovénisation » de la Carinthie, imaginée dans le passé comme une intrusion violente au sein du territoire, est maintenant décrite comme une infiltration silencieuse. M. Haider, chef du gouvernement de Carinthie, essaie en alternance de jouer de manière populiste les deux cartes. Tantôt il mène à l'encontre des pays voisins sa propre politique étrangère en contournant le pouvoir central de Vienne, tantôt il évoque les anciens préjugés et mobilise les sentiments populaires, comme ce fut le cas récemment dans la relance du conflit autour des panneaux topographiques bilingues. La mise en scène de ce conflit a clairement démontré que les « frontières dans la tête » sont encore loin d'être dépassées.

\section{Pas à pas, à travers la frontière ${ }^{5}$ : les efforts de la Coppla Kasa}

27 Malgré tout, les habitants d'Eisenkappel et des vallées de Vellach n'ont jamais cessé d'avoir des relations avec leurs voisins des villages slovènes de Jezersko et Solcava. Avant la Première Guerre mondiale, les trois communes font partie d'un même espace économique et administratif. Par exemple : au moment des gros travaux agricoles, la main d'œuvre circule d'un village à l'autre ; le bétail d'Eisenkappel monte en estive sur le versant sud de la Koschuta, qui constitue actuellement la frontière ; Jezersko dépend de la juridiction d'Eisenkappel; chaque automne les paysans de Solcava passent le col $\mathrm{du}$ Paulitsch avec leurs moutons pour les vendre sur la foire aux bestiaux d'Eisenkappel. Des deux côtés de la montagne, les habitants profitent de ces échanges multiples. Une fois la frontière tracée, d'un jour à l'autre le commerce légal avec les villes et villages voisins du sud n'est plus possible. La contrebande prend le relais (comme à l'époque de la route du sel) en fournissant un revenu aux nombreux chômeurs de l'entre-deux-guerres. On exploite les différences entre les nouvelles entités économiques, les produits trafiqués changent selon la conjoncture. La frontière gagne alors une aura d'interdit, de secret, d'illégal, échappant au contrôle des autorités.

Même dans la période où la frontière est fermée hermétiquement, les contacts interpersonnels se maintiennent grâce aux nombreux liens de parenté entre les familles des trois communes. De plus, la tradition de se réunir pour les fêtes religieuses dans les 
petites églises disséminées dans la forêt le long de la frontière a survécu à toutes les interdictions. Sur un plan plus organisé, l'association culturelle slovène d'Eisenkappel, Zarja, intensifie les échanges dans les années 60 après l'abolition des visas. Dans une première phase, ils sont limités à des manifestations culturelles ponctuelles comme des concerts, des représentations théâtrales, etc. Petit à petit, ils prennent une dimension plus importante en élargissant le spectre des activités aux familles entières. D'autres activités vont suivre, comme celles des chasseurs, des apiculteurs, et depuis quelques années des collèges d'Eisenkappel et de Preddvor.

Les échanges transfrontaliers pratiqués activement par toutes ces associations se passent en-dehors des programmes européens mis en place à cet effet. Seule l'association des paysans de montagne Coppla Kasa, avec ses cinquante fermes membres sur la commune d'Eisenkappel, a décidé d'emprunter la voie des démarches officielles. Ces petites et moyennes exploitations se sont regroupées depuis début 96 pour la vente en commun du bois et la mise en valeur de leurs produits traditionnels par la vente directe. Cette coopération non seulement doit permettre de maintenir un niveau de revenus garantissant aux paysans une vie digne sur leur ferme, et d'enrayer ainsi l'exode rural, mais elle signifie également, par le travail en commun, une revitalisation du tissu social des vallées. La Coppla Kasa s'est fait connaître hors de la région sur différents marchés et à l'occasion de sa fête annuelle, organisée en partenariat avec d'autres groupements paysans de vente directe en Carinthie du sud. Cette fête attire un public nombreux venu de tout le Land et de Slovénie.

Grâce à ces voyages dans les deux sens, les membres de Coppla Kasa découvrent les initiatives de leurs voisins des communes de Jezersko et Solcava. Situées comme Eisenkappel à la périphérie des états, elles sont confrontées aux mêmes problèmes: exode rural, manque d'emplois, grand nombre de résidents travaillant au-dehors. A Solcava, dernier village de la haute vallée de Savinja, les paysannes et les paysans ont réussi à renverser les tendances négatives. La vallée de Logar/Logarska dolina, sur la commune de Solcava, est un but d'excursions célèbre et apprécié en Slovénie. En 1987, elle a été classée parc naturel et mise sous protection pour prévenir une dégradation de l'environnement par le tourisme de masse. Les paysans et les propriétaires de la vallée ont constitué en 1992 une société de gestion du parc qu'ils gèrent eux-mêmes. Ils ont instauré à l'entrée un péage pour les voitures, l'accès étant gratuit pour les piétons. Les sommes encaissées permettent l'embauche de trois personnes pendant toute l'année. Elles ont la tâche de surveiller les lieux, d'entretenir les chemins de randonnée, d'aménager des aires de pique-nique, etc. Les visiteurs peuvent loger à l'hôtel ou sur la trentaine de fermes qui proposent des chambres d'hôtes.

31 Au moment où l'Autriche adhère à l'UE et où on prépare l'élargissement à l'Est, la coopération transfrontalière devient un thème important de l'agenda politique. L'UE lance un programme de financement destiné à sa promotion : le programme Interreg. Les membres de Coppla Kasa, désireux d'intensifier la coopération avec leurs voisins, élaborent avec eux le projet des «Chemins transfrontaliers de randonnée Eisenkappel-Jezersko-Solcava », qu'ils déposent dans le cadre d'Interreg. Dans la phase initiale du programme on constate que, pour l'Interreg Carinthie-Slovénie, il y a nettement moins de projets que pour l'Interreg Styrie-Slovénie. Un an avant la date limite de dépôt des dossiers pour Interreg II, fin 98, seule $25 \%$ de la somme destinée à l'agriculture a été demandée, et $10 \%$ pour le tourisme. Dans le volet agricole, en Carinthie, on trouve plusieurs initiatives similaires à celle de Coppla Kasa, comme par 
exemple un flock-book de la race de moutons locale, la relance de la culture du chanvre, un projet de formation agricole. Mais la plus grande tranche va finalement à des projets nécessitant des investissements lourds, comme l'achat de matériel agricole ou l'installation d'une station d'épuration. Finalement les fonctionnaires du gouvernement carinthien peuvent annoncer à la clôture d'Interreg II, fin octobre 2001, que la totalité de la somme a été dépensée. C'est en effet la condition pour que l'UE augmente le budget d'Interreg III. Même si le projet de Coppla Kasa, financièrement modeste, est cité comme exemplaire pour son caractère véritablement transfrontalier, pour la période actuelle d'Interreg III Carinthie-Slovénie (2001- 2006) seuls 3 projets associatifs ont été déposés jusqu'à maintenant dans l'agriculture. Ce recul sensible peut s'expliquer par un examen attentif de la genèse du projet des chemins de randonnée Eisenkappel-Jezersko-Solsava, qui montre bien la complexité du processus et la multitude des obstacles rencontrés.

Le projet de Coppla Kasa regroupe 22 fermes d'Eisenkappel-Vellach, 9 de Jezersko et 18 de Solcava. Il a pour buts :

1. le maintien de la fonction productive dans l'espace rural ;

2. la lutte contre l'exode rural ;

3. la liaison de l'agriculture à un tourisme respectueux de l'environnement ;

4. l'élargissement de l'offre de vacances à la ferme ;

5. la préservation de l'architecture paysanne ;

6. le renforcement de l'identité régionale transfrontalière ;

7. la coopération avec des initiatives sœurs à Solcava et Jezersko ;

8. la publication d'une carte des chemins de randonnée sur les trois communes.

Dans les années 1997 à 2000, plusieurs rencontres annuelles ont lieu successivement dans les trois communes, avec la participation des paysans concernés et de leurs familles. Petit à petit, en étudiant les cartes existantes, d'anciens chemins sont retrouvés, et les itinéraires des randonnées sont établis afin de passer par chacune des 49 fermes (fig. 1). Pour mieux connaître la situation dans l'autre pays, on organise de part et d'autre de la frontière des excursions chez les voisins, pour visiter leurs fermes. Les paysans slovènes sont curieux d'écouter les Autrichiens raconter leurs expériences après l'entrée dans l'UE, puisque la Slovénie ne saurait tarder. En attendant, la vente directe de leurs produits en Autriche et vice-versa n'est pas autorisée. Ce qui n'empêche pas les Slovènes de venir les faire déguster à la fête de Coppla Kasa. En retour, des membres de Coppla Kasa sont présents aux Journées du tourisme à Solcava. La présence des voisins est toujours très appréciée, aussi, dès la première année de la coopération, on envisage de créer un label commun aux trois communes.

Dès le début, le projet Interreg pose plusieurs problèmes. Même s'il est financé jusqu'à $80 \%$, un préfinancement est nécessaire. Une petite association comme Coppla Kasa n'a pas de ressources propres et ne fait pas de bénéfices. Les dépenses courantes sont couvertes par les cotisations de ses membres et les rentrées de la fête annuelle, elle n'a pas les moyens de s'endetter en prenant un crédit bancaire. Le préfinancement n'est donc possible que par les prestations facturées mais non-payées de ses membres. Une fois les factures déposées, il faut encore attendre pour le remboursement, car le gouvernement carinthien donne la priorité aux projets comportant de gros investissements et sans véritable fonction transfrontalière. La subvention pour les 
chemins de randonnée est accordée en juin 1997, les premières factures déposées en septembre 1999, et une première tranche est payée en avril 2001 seulement.

Ensuite, les exigences de l'administration, qui réclame que le projet se réalise au cours d'une année, ne tiennent pas compte des rythmes agricoles et des conditions géographiques et climatiques. Avant l'ouverture du col du Paulitsch en avril 2000, les communications entre les trois communes sont difficiles (par exemple, d'Eisenkappel à Solcava, la route fait un détour de $75 \mathrm{~km}$ ). Depuis, elles se sont améliorées, même si le col n'est ouvert que de mai à novembre.

Enfin, le projet se heurte à des problèmes juridiques. Dans l'accord passé en 1995 entre la République autrichienne et la jeune République slovène réglementant les randonnées transfrontalières en Carinthie, Styrie et Slovénie (Bundesgesetzblatt 1995), Eisenkappel n'est pas mentionné. L'association ne peut donc publier sa carte que si la loi est changée. Pour cela, la commune d'Eisenkappel doit déposer une motion auprès d'une commission paritaire austro-slovène qui se réunit une fois par an. D'abord la motion doit être agréée par les instances constitutionnelles, de la sécurité et des douanes des gouvernements des deux pays. Si la motion est acceptée par la commission, elle doit encore passer par les ministères de l'intérieur avant que l'amendement soit ratifié par les ministères des affaires étrangères. Puisque la commune reste inactive, les membres de Coppla Kasa eux-mêmes entreprennent en septembre 1999 ces démarches, qui n'aboutiront qu'au printemps 2002.

Entre-temps d'autres actions communes sont menées, comme la réalisation et la pose de panneaux pour les fermes d'Eisenkappel, dans le même style que ceux de Solcava, avec le sigle de la Kasa, mentionnant leur nom et leurs productions dans les deux langues. Pour faire connaître le projet à un maximum de gens, on a imaginé une publicité originale qui consiste en un gigantesque jeu de l'oie avec les itinéraires de la carte, présenté comme attraction dans les fêtes de village. En août 1999 le département de l'aménagement du territoire du gouvernement carinthien attribue un prix au projet dans le cadre d'un concours sur le thème " vivre en Carinthie ».

Les initiateurs du projet doivent recenser les cartes existantes. En Autriche et en Slovénie il existe des cartes topographiques à l'échelle de 1:50 000 avec des tracés de chemins de randonnée. Mais chaque État s'occupant uniquement de la mise à jour des données sur son territoire national. il n'existe pas de carte complète et actualisée transfrontalière. En outre, en Autriche, dans les cartes éditées par l'office fédéral de cartographie (Bundesamt für Eich- und Vermessungswesen, BEV) de Vienne, les noms topographiques et des lieux-dits slovènes sont germanisés, ce qui ne convient pas toujours aux usages locaux et aux habitants. L'office du tourisme d'Eisenkappel a édité en 1995 - un an après l'indépendance de la Slovénie - une carte de randonnées à l'échelle $1: 25.000$, mais le territoire slovène y est encore en blanc.

L'élaboration de la carte "Kasa Wege - Wandern über die Grenze » (Les chemins de la Kasa - randonnées pédestres transfrontalières) s'impose donc. Les initiateurs du projet font le tour des fermes concernées dans les trois communes pour consulter les habitants sur la manière dont ils souhaitent que soient écrits les noms propres. Le résultat est le plus souvent bilingue. Sur le dos de la carte sont présentés les produits et les services offerts dans chaque ferme, ainsi que les sites d'intérêt culturel.

La carte sort fin 2001. Elle éveille un écho dans les médias régionaux ainsi que dans des journaux de randonneurs en Suisse. A Solsava, Jezersko et Eisenkappel, le bilan est positif. Le chemin de randonnée ne relie pas seulement les trois communes entre 
elles, mais il attire un autre type de touristes, venant souvent des grandes villes, qui apprécie la qualité de l'accueil et des produits fermiers, ainsi qu'un environnement intact. Ute Logar, paysanne de Solcava, exprime son optimisme : "Chez nous, on attend des retombées économiques, avec la venue de nouveaux visiteurs; on espère aussi mieux connaitre nos voisins, peut-être nouer de nouvelles amitiés; en parcourant ensemble les chemins, pour nous, les frontières vont disparaître » (Émission télévisée « Kärnten heute », 31 août 2001).

41 Côté autrichien, au sein de Coppla Kasa, on considère que l'adhésion de la Slovénie à l'UE représente une chance pour le projet. Elle va permettre d'intensifier la coopération économique avec la commercialisation des produits fermiers sous un label de qualité commun. En attendant, la carte des chemins transfrontaliers a ravivé chez ses membres le sentiment de leur propre valeur, et la conscience de partager avec les voisins non seulement des problèmes, mais aussi des perspectives et des visions qui les rapprochent.

Le projet contribue au désenclavement des trois communes; pour faire revivre l'ancien espace économique et culturel, bien des frontières sont encore à abattre. Les initiatives associatives, qui regroupent des habitants des régions frontalières autour d'un projet concret, sont les véritables acteurs d'une coopération transfrontalière. Il serait souhaitable que les institutions concernées réservent dans leur programme un volet pour de tels projets associatifs, puisque ce sont d'abord les contacts personnels qui permettent de venir à bout des « frontières dans la tête ».

\section{BIBLIOGRAPHIE}

BALIBAR Étienne (1997). - La crainte des masses. Politique et philosophie avant et après Marx. Paris, Galilée.

BODENHÖFER Hans-Joachim (2000). - Kärnten und Slowenien. Wirtschaftliche Entwicklung 1920-2000. Universität Klagenfurt.

BUNDESGESETZBLATT für die Republik Österreich (1995) : Grenzübergänge für Wanderer und Bergsteiger in Kärnten, der Steiermark und Slowenien. Ausgegeben am 30.11.1995, 261. Stück, 778. Abkommen.

Busch Brigitta (1996). - Lepena. Ein Dorf macht Schule. Eine Mikrountersuchung sozialer und kultureller Gegensätze. Klagenfurt, Drava.

CoPpla KASA (ed). - Kasa-Wege, Wandern über die Grenze. Carte disponible à l'adresse suivante :

Heike Schiebeck, Lobnik 16, A-9135 Eisenkappel

EUROSTAT. - Ergebnisse der EU-Strukturerhebung 1997.

HeRzog Doris, WASTL-WALTER Doris (1995). - « The Economic Restructuring of Eisenkappel /

Zelezna Kapla - A Case Study of Sustainable Developement in an Underdeveloped Area ».

Klagenfurter geographische Schriften, $\mathrm{n}^{\circ} 13, \mathrm{pp}$. 126-147.

Revue Géographique de l'Est, vol. 43 / 4 | 2003 
HiPfl Brigitte, Bister Anita, STROHMAIER Petra, Busch Brigitta (2002). - « Shifting Borders : Spatial Constructions of Identity in an Austrian/Slovenian Border Region». Ulrike Meinhof (ed. ) : Living (with) Borders. Identity Discourses on East-West Borders in Europe. Aldershot, Ashgate Border region Series, p. 53-75.

Judson Pieter M. (2001). - « Frontier Germans : The Invention of the Sprachgrenze ». Susan Ingram, Markus Reisenleitner, Cornelia Szabo-Knotik (ed. ) : Identität, Kultur, Raum : kulturelle Praktiken und Ausbildung von Imagined Communities in Nordamerika und Zentraleuropa. Wien : Turia und Kant, p. 85-101.

KRAJASITS Cornelia (1998). - Zwischen Zentralraum und Grenze. Wirtschaftliche Entwicklungstendenzen in den österreichischen Grenzregionen. Wien, Österreichisches Institut für Raumplanung.

KRAMMER Josef (1996). - Österreichs EU-Beitritt : Die verpasste Chance für eine europaweite ökosoziale Agrarpolitik. Der kritische Agrarbericht. AgrarBündnis (ed.) Bonn.

Östat (1995). - Ein Blick auf die Gemeinde Eisenkappel-Vellach. Wien.

OstRAVsKY Miroslav (1968). - « Vom Handeslverkehr durch das obere Vellachtal. 700 Jahre Markt Eisenkappel », p. 40-52. Geschichtsverein für Kärnten [ed] Klagenfurt.

PUHovsкi Zarko (2000). - « Hate silence ». Nena Skopljanac Brunner ; Stjepan Gredelj ; Alija

Hodzic ; Branimir Kristofic : Media \& War. Zagreb, Centre for transition and civil society research and Belgrade, Agency Argument.

RECHBERG-OBIR (1995). - 100 Jahre Zellstoff-Rechberg-Obir - Die verlorene Zukunft. Rechberg-

Veteranen (ed).

SCHIEBECK Heike (2002). - Wandern über die Grenze. Eine humangeographische Fallstudie. Universität Klagenfurt, Institut für Geographie und Regionalforschung.

SHANIN Teodor (1990). - Defining Peasants : essays concerning rural societies, expolary economies, and learning from them in the contemporary world. Oxford, Basil Blackwell.

SHANIN Teodor. - Die Geheimnisse der Beziehungswirtschaft. Warum Russland trotzdem überlebt. Interview avec T. Shanin, WOZ 47/2000, Zürich.

STATISTK AUSTRIA, Volkszählung 1981; Volkszählung 1991 ; Volkszählung 2001.

Agrarstrakturerhebung 1999, Gesamtergebnisse. www.statistik.at.

ToDorova Maria (1999). - Die Erfindung des Balkans. Europas bequemes Vorurteil. Darmstadt, Primus Verlag.

VALENTIN Hellwig (1997). — « Eine schwierige Nachbarschaft. Die Beziehungen zwischen Kärnten und Slowenien mit besonderer Berücksichtigung der Jahre 1945 bis 1995 ». Karl Anderwald ; Peter Karpf ; Hellwig Valentin : Kärntner für Politik. Klagenfurt Kärntener Druck und Verlagsgesellschaft.

VeliKonja Mitja (1996). - Masade duha. Razpotja sodobnih mitoloij. Ljubljana, Zbirka Sophia.

WODAK Ruth, DE CILLIA Rudolf, REISIGL Martin, LIEBHART Karin (1999). - The discursive construction of national identity. Edinburgh University Press.

\section{NOTES}

1. Fer, en allemand Eisen, d'où le nom Eisenkappel 
2. Les chiffres du recensement de 2001 ne sont pas encore disponibles dans tous les domaines à la date de rédaction de cet article.

3. Toutes les traductions des citations sont des auteurs de l'article.

4. Coppla : de l'ancien nom d'Eisenkappel, et Kasa (prononcer « cacha ») : nom slovène donné à la petite maison en bois dans laquelle les paysans gardent leurs provisions et sèchent la charcuterie (Fig. 3).

5. Cette partie de notre contribution est basée sur une thèse acceptée par l'Institut de géographie et de recherche régionale, Université de Klagenfurt (Schiebeck 2002).

\section{RÉSUMÉS}

Bien que la coopération transfrontalière soit aujourd'hui à l'ordre du jour, avec l'élargissement de l'UE à l'Est, elle se heurte à de nombreux obstacles. L'article traite des communes frontalières d'Eisenkappel-Vellach /Zelezna kapla Bela, Jezersko et Solcava. Après une description des caractéristiques démographiques et socio-économiques de ces espaces périphériques, il analyse dans une perspective historique les réticences mentales et la construction des mythes autour de la frontière, qui continuent à empêcher le développement de l'espace transfrontalier en Carinthie. L'étude d'un projet du programme Interreg Carinthie-Slovénie montre comment de petites initiatives associatives ont réussi à réaliser leur coopération transfrontalière.

Despite the fact that cross-border cooperation will be promoted in many ways by EU enlargement, there are still many obstacles in its path. The article focuses on the border municipalities of Eisenkappel-Vellach/_elezna kapla Bela, Jezersko et Sol_ava. It starts with a sketch of the demographic and socio-economic characteristics of this peripheral zone. The article then goes on to analyse within a historic perspective the mental reservations and the creation of myths around the frontier which in Carinthia continue to represent an obstacle to the development of the border region. In contrast, the case study of a project carried out within the framework of the EU Interreg programme shows how bottom-up initiatives can be developed on the basis of cross-border cooperation.

Mit der bevorstehenden EU-Osterweiterung wird die grenzüberschreitende Zusammenarbeit zwar auf vielfältige Weise gefördert, stößt jedoch auf Hindernisse. Der vorliegende Artikel fokussiert auf die Grenzgemeinden Eisenkappel-Vellach / Zelezna kapla-Bela, Jezersko und Solcava. Nach einer demografischen und sozioökonomischen Skizze der Charakteristika dieses peripheren Raumes, analysiert er in einer historischen Perspektive mentale Vorbehalte und Mythenbildungen rund um die Grenze, die in Kärnten nach wie vor ein Hindernis für die Entwicklung des Grenzraumes darstellen. Demgegenüber zeigt die Fallstudie eines Projektes im EU-Programm Interreg Kärnten-Slowenien, wie Bottom-up-Initiativen die Chancen grenzüberschreitender Zusammenarbeit dennoch für sich nutzen. 
INDEX

Mots-clés : coopération transfrontalière, élargissement de l'UE, espace frontalier CarinthieSlovénie, mythes autour de la frontière, programme Interreg

Schlüsselwörter : EU-Osterweiterung, Grenzgebiet Kärnten-Slowenien, Grenzmythen, grenzüberschreitende Zusammenarbeit, Interreg-Programm

Keywords : border myths, border space Carinthia-Slovenia, cross-border cooperation, EUenlargement, Interreg Programme

\section{AUTEURS}

\section{BRIGITTA BUSCH}

Université de Klagenfurt et Université de Vienne - Karawankenblickstrasse 215 - A-9020

Klagenfurt - brigitta.busch@uni-klu.ac.at

\section{HEIKE SCHIEBECK}

Lobnik 16 - A-9135 Eisenkappel/Zelezna kapla - stopar@aon.at 